\title{
La gestión de la información en las empresas de Extremadura
}

\author{
Antonio Muñoz-Cañavate*, Antonio Pulgarín-Guerrero*
}

Resumen: Este trabajo presenta los resultados más relevantes de una encuesta realizada a las empresas con sede social en Extremadura y con mayor número de empleados y facturación. El objetivo principal ha sido conocer el comportamiento empresarial en la gestión de información, los usos y necesidades informativas de las empresas extremeñas, y especialmente la información que se encuentra en el entorno. La metodología ha consistido en el envío y posterior recogida de un cuestionario a 144 empresas seleccionadas, de las cuales respondieron 73. Sólo cinco de las empresas que respondieron tenían más de 250 empleados, sin embargo 23 de esas empresas tenían una facturación superior a los 10.000.000 de euros. La encuesta final es el resultado de un cuestionario piloto que se envió a varias a empresas y de las sugerencias aportadas por distintos expertos españoles antes de su envío definitivo.

Un 69,86\% no disponía de ningún tipo de unidad de información y/o documentación, pero un 46,58\% sí disponía de algún mecanismo estructurado encargado de capturar el conocimiento del entorno para la toma de decisiones. Destaca la mayor importancia como canal de captura de información que se da a la información procedente del denominado medio ambiente externo inmediato, como la información que llega de distribuidores, proveedores y clientes. Y en cuanto al tipo de institución de la que más información recibe la empresa para tomar decisiones, sin duda las asociaciones de empresarios son las que mayor porcentaje alcanzan (más del 60\%), seguido de las entidades financieras y de la administración regional.

Palabras clave: Extremadura, empresas, gestión de la información, entorno, inteligencia competitiva.

\section{Information management by companies in Extremadura}

Abstract: This paper presents the most important results of a survey of the largest companies - in terms of number of employees and turnover - with their headquarters in Extremadura (Spain). The main objective was to determine corporate behaviour with respect to information management, the need for information and its uses, and the information collected from the companies business environment. A questionnaire was sent to 144

* Universidad de Extremadura. Correo-e: amunoz@alcazaba.unex.es; apulgue@alcazaba.unex.es Recibido: 4-11-2008; 2. ${ }^{\text {a }}$ versión: 23-12-2008. 
selected companies, of which 73 responded. Only 5 of the responding companies had more than 250 employees, but 23 had a turnover higher than 10,000,000 Euros. The final survey questions reflected the results of a pilot questionnaire sent to a smaller number of companies and the suggestions made by various Spanish experts. Some $69.86 \%$ of respondents had no form of information and/or library services unit, although $46.58 \%$ did have some structured mechanism responsible for capturing knowledge for decision-making from the business environment. Information from distributors, suppliers and clients was regarded as the most important information capturing channel. Trade associations were the most important bodies providing information for decision-making (>60\%), followed by financial institutions and the regional authorities.

Keywords: Extremadura, business, information management, environment, competitive intelligence.

\section{Introducción}

Que duda cabe que los instrumentos y las fuentes de información para la empresa han mejorado en España durante los últimos años, y ello debido a que la inserción de Internet ha permitido que multitud de agentes públicos y privados hayan podido poner en marcha herramientas informativas para las empresas o, en otros casos, transformar las que ya tenían antes de la generalización de la Red a mediados de los años noventa.

Glynn y Koening (1995) analizan el dramático cambio experimentado en el entorno informativo de las pymes que definían en la aparición de los sistemas electrónicos de recuperación de información, en los brockers de información, y en la adaptación como proveedores de información para la empresa de las propias Administraciones Públicas o incluso de las bibliotecas.

Es evidente que en España ha cambiado sobremanera el entorno de la información para la empresa desde aquel Sistema de Información Empresarial (SIE) puesto en marcha por el Instituto de la Pequeña y Mediana Empresa Industrial (IMPI) en colaboración con las comunidades autónomas en el que se partía de una base muy simple pero que no siempre se había explicitado, y es que en la moderna gestión empresarial la información se había convertido en un elemento básico para garantizar su eficacia (Zamorano Martínez, 2005).

A las bases de datos de organismos oficiales como el IMPI (en la actualidad DGPYME), el Instituto Español de Comercio Exterior (ICEX), la Oficina Española de Patentes y Marcas (OEPM), la aparición de Camerdata, o las experiencias de algunas entidades financieras, le seguiría una incipiente industria de la información electrónica en el sector privado y en ámbitos muy diversos como la información legislativa y jurídica, o la información comercial.

La generalización y expansión de Internet permitió que esos productos informativos aumentaran el rango potencial de su clientela a la vez que mejoraban sus interfaces de consulta y recuperación de información.

Internet tuvo, además, otro efecto como fue el de mejorar las herramientas de comunicación y de transferencia de información de medios de comunicación, cámaras de comercio, asociaciones empresariales y organismos de soporte a la I+D+I. 
De manera que con el tiempo se ha construido un conglomerado de fuentes de información.

Así, el estudio de la gestión de la información empresarial puede ser analizado desde distintas perspectivas, como, por ejemplo, desde el complejo de recursos informativos existente para la empresa, o desde el consumo y uso que las empresas hacen de la información.

Los estudios acerca de los usos informativos de las empresas españolas no son numerosos en el campo de la información y documentación en España, tampoco lo es la presencia de esta disciplina en las titulaciones de Documentación en el país (Muñoz Cañavate, 2006; 2007; 2008). Entre los estudios realizados en España encontramos algunos realizados para las empresas de la Comunidad de Madrid (Giménez Toledo, 2004) (Vázquez Valero, 1999), en las empresas exportadoras (Postigo, 2001), en las multinacionales catalanas (Tena Millán, 2004), o los publicados por el Global Market Intelligence Study en el que intervienen empresas españolas (GIA International, 2005 y 2007).

\section{Objetivos}

Este trabajo ha tenido como objetivo general el conocimiento de las prácticas de gestión de información de las empresas con mayor facturación y número de empleados de Extremadura. Los objetivos específicos se han centrado en conocer la existencia de unidades de información y documentación en las empresas y la presencia de personal dedicado a las tareas de recopilación, organización y análisis de la información. También la existencia de modos o prácticas de gestión del conocimiento, y el uso de la variada tipología de información que cualquier compañía tiene a su disposición.

Además, y respecto a la procedencia de la información que se utiliza para la toma de decisiones cuyo origen es el medio ambiente externo, la encuesta aplicada ha diferenciado entre la importancia del entorno remoto (que se estructura en los ámbitos socio-cultural, económico, tecnológico, político y legal), y el entorno inmediato (en el que se encuentran los competidores, los proveedores, los clientes y las Administraciones Públicas).

Finalmente, el interés de los autores ha buscado conocer el origen concreto de la información que se utiliza para tomar decisiones distinguiendo entre información que llega a través de documentos (en papel o soporte electrónico) y los canales informales (como las conversaciones). Todo ello buscando la representatividad de los organismos, instituciones y personas de las que procede la información, ya sea ésta formal o informal.

\section{El entorno. La empresa extremeña}

Extremadura cuenta con una población total de 1.089.990 habitantes (según INE a 1 de enero de 2007) lo que representa el 2,4\% de la población de España. 
En 2007 disponía según el DIRCE de 66.232 empresas (el 1,9\% del total nacional). Sobre el total nacional la provincia de Badajoz tenía el 1,2\% de las empresas, mientras a la provincia de Cáceres le correspondía el 0,7\%.

La estructura de las empresas requiere una descripción aparte ya que son mayoritarias las empresas con pocos asalariados. Así, de las más de 40.000 empresas de la provincia de Badajoz, sólo 239 tenían más de 50 asalariados (el 0,5\%), mientras de las más de 26.000 de la provincia cacereña la cifra se reducía a 119 (el $0,4 \%$ del total).

Más bajas son las cifras que señalan los gastos internos totales y personal en $\mathrm{I}+\mathrm{D}$, ya que Extremadura representa el $1 \%$ de los gastos internos y el $1 \%$ del personal de I+D, respecto al total nacional (INE, 2006).

\section{Metodología}

Para la realización de este estudio se confeccionó un cuestionario (véase anexo 1), que fue previamente enviado en el período de diseño a varios expertos españoles en Inteligencia Competitiva, además de ser validado en varias empresas, antes del envío definitivo, que permitió mejorar la presentación de los distintos ítems. Es necesario aclarar que los resultados totales son más que los que se presentan en este artículo. Se han seleccionado aquí, por tanto, los que los autores consideran más importantes. Este cuestionario se envió a los gerentes y/o administradores de las empresas que se habían seleccionado para la muestra, y fueron ellos los que respondieron. Se decidió utilizar como muestra a las 150 empresas con mayor volumen de facturación y mayor número de empleados de la región (lo que permitía realizar el estudio a empresas con una mínima estructura organizativa), para lo cual se utilizó la base de datos ARDAN. En todos los casos eran empresas con sede social en la región, lo que garantizaba obtener resultados sobre la cultura empresarial y los modos de gestión de la información del entorno extremeño. De la muestra inicial quedaron 144 empresas, una vez que fueron descartadas algunas firmas, ya que varias habían dejado de existir y otras eran sociedades del mismo propietario y sector que otras empresas de la muestra y fueron unificadas en un único cuestionario. De las 144 empresas finalmente seleccionadas, 109 correspondían a tres sectores (códigos CNAE): construcción y contratas, fabricantes y mayoristas, tal y como puede comprobarse en la Tabla I.

Es necesario destacar que, por las opiniones expresadas durante el proceso de recogida de datos, las empresas son entidades poco dadas a responder encuestas y más todavía cuando hacen referencia a asuntos de gestión interna muy particulares (durante el proceso algunas empresas declinaron contestar debido a que según sus gestores se hacía mención a cuestiones privadas). Algunos de los evaluadores previos de la encuesta insistieron en los escasos resultados que podríamos conseguir si no ofrecíamos un cuestionario lo más sencillo posible y con preguntas cerradas, que pudiera responderse lo más brevemente posible. Uno 
TABLA I

Empresas por sectores que forman parte de la encuesta y total de respuestas

\begin{tabular}{l|c|c|c}
\hline \multicolumn{1}{c|}{ Sectores } & $\begin{array}{c}\text { Empresas } \\
\text { encuestadas }\end{array}$ & $\begin{array}{c}\text { Empresas } \\
\text { que } \\
\text { responden }\end{array}$ & $\begin{array}{c}\text { \% de } \\
\text { respuesta }\end{array}$ \\
\hline 01-09 Agricultura, silvicultura, ganadería y pesca & 7 & 3 & 42,86 \\
\hline 10-14 Minería y explotación & 1 & 1 & 100,00 \\
\hline 15-17 Construcciones y contratas & 44 & 21 & 47,73 \\
\hline 20-39 Fabricantes & 39 & 20 & 51,28 \\
\hline $\begin{array}{l}\text { 40-49 Transportes, comunicaciones y servicios } \\
\text { públicos }\end{array}$ & 5 & 2 & 40,00 \\
\hline 50-51 Mayoristas & 26 & 14 & 53,85 \\
\hline 52-59 Detallistas & 6 & 4 & 66,67 \\
\hline 70-89 Servicios & 15 & 8 & 53,33 \\
\hline 91-93 Organismos oficiales & 144 & 73 & 0,00 \\
\hline Total & & 50,69 \\
\hline
\end{tabular}

de los expertos nos señaló, respecto a la primera versión del cuestionario, que se pedían datos que podían resultar parte de la estrategia de las empresas y que una vez analizados podían sacar a la luz debilidades y convertirse en amenaza si se conocía esa información. También es necesario destacar que en la carta inicial que acompañaba a la encuesta se decía que todo el procesamiento de los datos sería anónimo y que se tratarían con absoluta discreción y reserva, obteniéndose datos globales para el conjunto de las empresas, nunca de forma individual.

Para enviar las encuestas a las empresas se utilizaron dos métodos: $a$ ) entrega y recogida personal, esta fue la primera forma de distribución, y b) el envío y recepción por correo postal, en segundo lugar. En ningún caso se utilizó el correo electrónico para el envío de la encuesta.

Sin duda la ayuda de un numeroso grupo de alumnos de distintas titulaciones de la Universidad de Extremadura permitió la entrega y recogida personal en las empresas distribuidas tanto en las dos capitales provinciales y la capital autonómica, como en un gran número de municipios de la región.

En todos los casos la encuesta se acompañaba de una carta de presentación, precedida, en el caso de los envíos realizados por correo postal, de una llamada de teléfono.

La Tabla II muestra el índice de respuesta de los dos procedimientos, algo superior en el caso de la entrega y recogida personal $(51,64 \%)$ frente al sistema que utilizó el correo postal (45,45\%). 


\section{TABLA II}

Método de encuesta

\begin{tabular}{l|c|c}
\hline & $\begin{array}{c}\text { Entrega y recogida } \\
\text { personal }\end{array}$ & $\begin{array}{c}\text { Envio y recepción } \\
\text { por correo }\end{array}$ \\
\hline Total & 122 & 22 \\
\hline Contestan & 63 & 10 \\
\hline No contestan & 59 & 12 \\
\hline \% de respuesta & 51,64 & 45,45 \\
\hline
\end{tabular}

Hay que señalar que de las empresas que respondieron la mayoría tenía acceso a Internet (71 de las encuestadas), y solo dos no tenían conexión. Además, 44 empresas disponían de web corporativo.

Sobre el número de empleados de las 73 empresas, cinco tenían menos de 50 empleados, 46 empresas disponían de entre 50 y 100 empleados, 17 empresas se encontraban en la franja que va de 101 a 249 empleados y sólo cinco empresas tenían más de 250 empleados.

Y sobre la facturación, las empresas que respondieron se encontraban en las siguientes franjas: 14 con una facturación anual entre 600.000 y 3.000 .000 de euros; 36 empresas con una facturación entre 3.000.000 y 10.000.000 de euros; y 23 empresas con una facturación superior a los 10.000.000 de euros.

\section{Resultados y discusión}

\subsection{El profesional de la información y las unidades de información en las empresas}

Los resultados de este trabajo señalan una escasa penetración del profesional de la información en las plantillas de las empresas. Los autores de ese trabajo piensan que tal vez no se le ha dado a la figura del documentalista de empresa, gestor de la información empresarial o vigilante del entorno, la importancia que debiera en el ámbito académico español, y nos referimos expresamente a las titulaciones de documentación y sus planes de estudio (Muñoz-Cañavate, 2007; 2008). Aunque en el caso de España también se cita como razón la que aduce que el documentalista está más preocupado por sus procesos internos que en servir de ayuda real a los objetivos estratégicos de la empresa (Fernández Bajón, 1998).

Se han de destacar en este sentido, los trabajos realizados por la ADBS en Francia. En el Référentiel des métiers-types des professionels de líinformation-documentation publicado en 2001 se cita, por ejemplo, el métier-type 19 "vigilantedocumentalista", que se encarga de la vigilancia estratégica y de la búsqueda de información muy diversa, enfocada a suministrar información a los equipos di- 
rectivos sobre la evolución del entorno. Esta figura emergente del responsable de inteligencia competitiva o económica también ha sido resaltada en 2006 por otras asociaciones y organismos además de la ADBS, y nos referimos a la ACFCI, ANFE, FEPIE, HRIE, IFIE, SCIP Francia.

Esta figura puede detectar fuentes adecuadas a las necesidades de la empresa generando valor añadido a la organización (Gold, 1999).

La Tabla III refleja, para nuestro trabajo, el resultado de la existencia de biblioteca o unidad de información en la empresa, lo cual no tiene necesariamente que identificarse con las unidades administrativas especializadas en el conocimiento y análisis del entorno que ya han sido tratadas, en la literatura sobre gestión de la información, desde hace décadas (Lenz, 1986). Desde el punto de vista práctico, tal vez debiera desterrarse de la empresa la idea de biblioteca o unidad de documentación frente a un concepto más amplio de unidades dedicadas a conocer el entorno para la toma de decisiones.

TABLA III

Número de empresas que tenían biblioteca y/o unidad de información/ documentación

\begin{tabular}{l|c|c}
\hline \multicolumn{1}{c|}{$(\boldsymbol{n}=\mathbf{7 3 )}$} & $\boldsymbol{f}_{\boldsymbol{i}}$ & $\boldsymbol{\%}$ \\
\hline Sí & 20 & 27,40 \\
\hline No & 51 & 69,86 \\
\hline No contesta & 2 & 2,74 \\
\hline
\end{tabular}

En nuestro estudio más del 69\% de las empresas no disponían de biblioteca o unidades de información y/o documentación. Además, se interrogó acerca de la existencia de personas dedicadas a la gestión de información (en concreto se señalaban las tareas de recopilación, organización y análisis de información relevante para la empresa). En la pregunta se hacía distinción entre las personas que no tenían dedicación exclusiva y las que si la tenían (Tabla IV). Los resultados muestran que el $45,21 \%$ de las empresas disponían de personal sin exclusividad a este tipo de labores, aunque se reducía drásticamente a un 2,74\% (dos empresas) si la pregunta se refería a la exclusividad.

Además, con otra pregunta (Tabla V) se trataba de corroborar, en cierta medida, las cuestiones anteriores. La pregunta en cuestión fue: ¿dispone de algún mecanismo estructurado (departamento o persona) encargado de capturar información externa para la toma de decisiones? El resultado es elocuente ya que las respuestas afirmativas representan un 46,58\% de las empresas, dato casi idéntico al de la Tabla IV, cuando se hacía referencia a la no exclusividad de las tareas de gestión de la información. 


\section{TABLA IV}

Número de empresas que tenían personal o unidad dedicado a tareas de recopilación, organización y análisis de información relevante para la empresa, tanto con dedicación exclusiva o no

\begin{tabular}{l|l|c|c}
\hline \multicolumn{2}{c|}{$(\boldsymbol{n}=\mathbf{7 3})$} & $\boldsymbol{f}_{\boldsymbol{i}}$ & $\mathbf{\%}$ \\
\hline \multirow{2}{*}{$\begin{array}{l}\text { Sin dedicación ex- } \\
\text { clusiva }\end{array}$} & $\mathbf{S i ́}$ & 33 & 45,21 \\
\cline { 2 - 4 } & No & 40 & 54,79 \\
\hline \multirow{2}{*}{$\begin{array}{l}\text { Con dedicación ex- } \\
\text { clusiva }\end{array}$} & Sí & 2 & 2,74 \\
\cline { 2 - 4 } & No & 68 & 93,15 \\
\cline { 2 - 4 } & No contesta & 3 & 4,11 \\
\hline
\end{tabular}

TABLA V

Número de empresas que tenían personal o unidad encargado de capturar información externa para la toma de decisiones

\begin{tabular}{l|c|c}
\hline \multicolumn{1}{c|}{$(\boldsymbol{n}=\mathbf{7 3})$} & $\boldsymbol{f}_{\boldsymbol{i}}$ & $\boldsymbol{\%}$ \\
\hline Sí & 34 & 46,58 \\
\hline No & 34 & 46,58 \\
\hline No contesta & 5 & 6,85 \\
\hline
\end{tabular}

Podemos decir que se vislumbra un cierto interés en capturar la información del exterior (afecta sólo a la mitad de las empresas), aunque esta actividad no se hace mayoritariamente con personas dedicadas en exclusividad a ello.

\subsection{La gestión del conocimiento}

La Tabla VI presenta los resultados de dos preguntas distintas pero orientadas a conocer la existencia de alguna forma de gestión del conocimiento. Llegados a este punto es importante resaltar que el concepto gestión del conocimiento conlleva una alta dosis de ambigüedad y desconocimiento para el mundo de la empresa, y, desde luego, más todavía para aquellas empresas que aún teniendo una cierta envergadura por el número de empleados, y consecuentemente disponen de una mínima estructura organizativa, no disponen de departamentos y políticas específicas al respecto. De esta manera las preguntas sobre gestión del conocimiento no deben de realizarse directamente, es decir, no se debe preguntar si la compañía en cuestión realiza labores de gestión del conocimiento, ya que esta cuestión podría ser interpretada de múltiples maneras y ello invalidaría 


\section{TABLA VI}

Número de empresas con práctica en la gestión del conocimiento

\begin{tabular}{|c|c|c|c|}
\hline \multicolumn{2}{|l|}{$(n=73)$} & $f_{i}$ & $\%$ \\
\hline \multirow{3}{*}{$\begin{array}{l}\text { ¿Dispone de alguna forma estructurada } \\
\text { para beneficiarse y registrar en una me- } \\
\text { moria de la empresa, los conocimientos } \\
\text { de los empleados? }\end{array}$} & Sí & 26 & 35,62 \\
\hline & No & 40 & 54,79 \\
\hline & No contesta & 7 & 9,59 \\
\hline \multirow{3}{*}{$\begin{array}{l}\text { Si sus empleados tienen información so- } \\
\text { bre sus competidores ¿existe algún me- } \\
\text { dio, en su organización, para compartir } \\
\text { esa información? }\end{array}$} & Sí & 23 & 31,51 \\
\hline & No & 43 & 58,90 \\
\hline & No contesta & 7 & 9,59 \\
\hline
\end{tabular}

las respuestas. En los cuestionarios estas preguntas deben especificar qué se entiende por prácticas concretas de gestión del conocimiento, lo que permite garantizar que el encuestado ha entendido lo que se pregunta y, por tanto, las respuestas son homogéneas.

En nuestro estudio, y tal y como se puede comprobar en la tabla correspondiente entre un 30 y $35 \%$ de las empresas habían implementado alguna práctica al respecto.

\subsection{Las fuentes de información impresas o en soporte electrónico}

A su vez, en un intento de estructurar los recursos de información en función de su contenido (datos que pueden proceder tanto de organismos públicos como privados), y no del ente del que emanan, se realizó la clasificación que se incluye en la Tabla VII.

Si observamos los resultados de la columna «al menos una vez en el últimos mes" encontramos que la prensa es la fuente más valorada con un 52,05\% de las compañías encuestadas, seguida de catálogos productos y/o catálogos de ferias comerciales (46,58\%), y con el mismo porcentaje la legislación, jurisprudencia y normativas entre los que se incluyen los convenios colectivos.

En un siguiente nivel, con cifras que se encuentran en torno al 39\% aparecen las revistas y la información sobre ayudas y concursos públicos.

Entre la franja porcentual que va del 30\% al 35\% se encuentra una amplia variedad de documentos que hemos insertado bajo el apartado «indicadores del entorno" en el que se incluyen las estadísticas sobre microeconomía y macroeconomía, y los informes económicos realizados por organismos muy diversos desde entidades financieras a Administraciones; también la información sobre los mercados financieros; y las fuentes que hacen referencia al apartado direcciones de empresas, productos e informes comerciales. Sin embargo esta última tipología de documento se encuentra, a su vez, entre las que "rara vez o nunca" han 


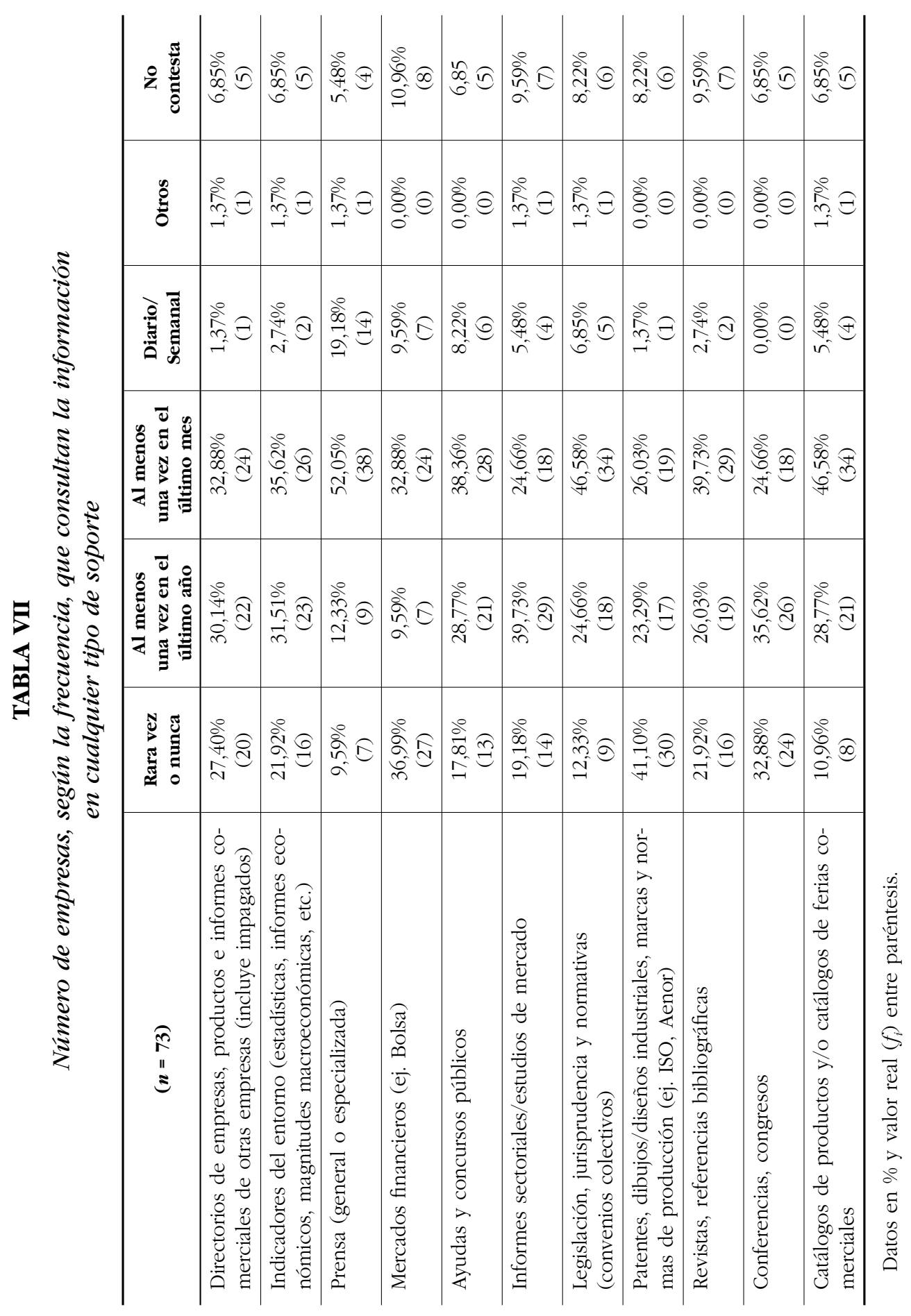


sido utilizadas por las empresas con un 27,40\%. Dato que debe de hacer reflexionar si tenemos en cuenta que un informe comercial presenta una radiografía muy completa de cualquier compañía.

Finalmente la información tecnológica, o los informes sectoriales y los estudios de mercado aparecen entre los menos demandados. En el caso de la información tecnológica su escasa incidencia entre las empresas extremeñas queda corroborada porque además existe un $41,10 \%$ de empresas que rara vez o nunca usan la información de patentes, diseños y dibujos industriales, marcas y normas de producción, lo cual tampoco debe extrañar ya que en la muestra no se caracteriza por la presencia de empresas de base tecnológica, aquellas productoras de bienes y servicios con un valor añadido en conocimiento, donde la investigación y el desarrollo juegan un papel fundamental.

\subsection{Procedencia de la información}

El conocimiento del entorno ha conformado tradicionalmente una parte importante en la gestión empresarial. Es obvio que en los procesos de planificación estratégica se requiere de información acerca de aspectos muy dispares que van desde las necesidades de los clientes al conocimiento de la situación política de un lugar determinado. Aquí la dimensión externa adquiere un papel fundamental.

Se consideran a Aguilar (1967) y a Keegan (1968), como algunos de los más destacados precursores de esta visión externa. Desde aquellos primeros autores se han sucedido los trabajos que tratan, entre otros aspectos, el proceso técnico de análisis del entorno aplicado a la estrategia empresarial, o la aplicación de ese proceso a sectores concretos.

Toda empresa, como toda organización puede ser analizada desde dos visiones, la visión interna y la visión externa, y aunque entendemos que las dos son importantes, existen defensores de anteponer a una por delante de la otra. Así, mientras los valedores de la visión interna entienden que los recursos (tangibles o intangibles) juegan un rol más importante que los elementos que se encuentran en el exterior, los defensores de la visión externa piensan que el entorno exterior es la fuente de beneficio para la organización (Tena Millán, 2006).

Se entiende el medio ambiente externo como todos aquellos factores que pueden afectar directa o indirectamente de manera perceptible a la empresa (Ivancevich, 1997). A su vez existe unanimidad en estructurar este ámbito externo en dos: uno más cercano al núcleo de gestión (entorno inmediato), o a la misma empresa en toda su extensión, como los clientes, competidores, proveedores, empleados, distribuidores, etc.; y otro más lejano (entorno remoto) cuyos cambios también afectan al funcionamiento de la organización. En este caso nos referimos a los ámbitos económico, cultural, tecnológico, político, legal, cuyas alteraciones afectan a la propia organización.

Los estudios de Xianzhong, Roland y Duan (2003), señalan que los directivos británicos perciben la información del entorno inmediato como más importante 
desde el punto de vista estratégico que la información del entorno lejano. El estudio de Auster y Choo (1994), sobre los sectores de la edición y las telecomunicaciones, incide en que los ejecutivos dan más importancia a la información sobre competidores, clientes, las normas reguladoras y los sectores tecnológicos, dando menos importancia a los ámbitos económico y sociocultural.

Las Tablas VIII y IX muestran los resultados de la encuesta acerca del uso de la información procedente del medio ambiente externo remoto e inmediato, respectivamente.

\section{TABLA VIII}

Distribución del número de empresas, según la frecuencia, que usan la información procedente del medio ambiente externo (Entorno remoto)

\begin{tabular}{|c|c|c|c|c|c|c|c|}
\hline \multicolumn{2}{|l|}{$(n=73)$} & \multirow{2}{*}{$\begin{array}{c}\text { Rara vez } \\
\text { o nunca }\end{array}$} & \multirow{2}{*}{$\begin{array}{c}\text { Al menos } \\
\text { una vez } \\
\text { en el } \\
\text { último } \\
\text { año }\end{array}$} & \multirow{2}{*}{$\begin{array}{c}\text { Al menos } \\
\text { una vez } \\
\text { en el } \\
\text { último } \\
\text { mes }\end{array}$} & \multirow{2}{*}{$\begin{array}{c}\text { Diario/ } \\
\text { Semanal } \\
0\end{array}$} & \multirow{2}{*}{$\begin{array}{c}\text { Otros } \\
0\end{array}$} & \multirow{2}{*}{$\begin{array}{c}\begin{array}{c}\text { No } \\
\text { contesta }\end{array} \\
1\end{array}$} \\
\hline Sociedad y cultura & $f_{i}$ & & & & & & \\
\hline Socheda y cuitua & $\%$ & 80,82 & 9,59 & 8,22 & 0,00 & 0,00 & 1,37 \\
\hline \multirow{2}{*}{ Economía } & $f_{i}$ & 6 & 29 & 33 & 4 & 0 & 1 \\
\hline & $\%$ & 8,22 & 39,73 & 45,21 & 5,48 & 0,00 & 1,37 \\
\hline \multirow{2}{*}{ Tecnología } & $f_{i}$ & 7 & 20 & 40 & 2 & 0 & 4 \\
\hline & $\%$ & 9,59 & 27,40 & 54,79 & 2,74 & 0,00 & 5,48 \\
\hline \multirow{2}{*}{ Política, legislación } & $f_{i}$ & 6 & 20 & 39 & 4 & 1 & 3 \\
\hline & $\%$ & 8,22 & 27,40 & 53,42 & 5,48 & 1,37 & 4,11 \\
\hline
\end{tabular}

En la información procedente del entorno remoto predomina la procedencia del ámbito económico, político y de legislación; cuando se refiere "al menos una vez al mes". La procedente del entorno inmediato es, también, la de "al menos una vez al mes", la más proclive a utilizarse.

\subsection{Organismos y entidades utilizados para tomar decisiones}

La Tabla X muestra el uso de la procedencia directa de las fuentes según estén en formato papel o en soporte electrónico, y en este segundo caso la pregunta incluía tanto los soportes en discos (ej. CdRom) como en Internet. Si sumamos las opciones "diario/semanal" y "al menos una vez en el último mes", que pensamos representan un uso continuado de las fuentes, podemos observar como destaca la opción "Asociación empresarial sectorial" como la más valorada con 


\section{TABLA IX}

Distribución del número de empresas, según la frecuencia, que usan la información procedente del medio ambiente externo (Entorno inmediato)

\begin{tabular}{|c|c|c|c|c|c|c|c|}
\hline \multicolumn{2}{|l|}{$(n=73)$} & \multirow{2}{*}{$\begin{array}{c}\text { Rara vez } \\
\text { o nunca }\end{array}$} & \multirow{2}{*}{$\begin{array}{c}\begin{array}{c}\text { Al menos } \\
\text { una vez } \\
\text { en el } \\
\text { ulltimo } \\
\text { año }\end{array} \\
20 \\
\end{array}$} & \multirow{2}{*}{$\begin{array}{c}\text { Al menos } \\
\text { una vez } \\
\text { en el } \\
\begin{array}{c}\text { último } \\
\text { mes }\end{array} \\
28 \\
\end{array}$} & \multirow{2}{*}{$\begin{array}{c}\text { Diario/ } \\
\text { Semanal } \\
4\end{array}$} & \multirow{2}{*}{$\begin{array}{c}\text { Otros } \\
1 \\
\end{array}$} & \multirow{2}{*}{$\begin{array}{c}\begin{array}{c}\text { No } \\
\text { contesta }\end{array} \\
2 \\
\end{array}$} \\
\hline Competidores & $f_{i}$ & & & & & & \\
\hline Compeciutises & $\%$ & $24,66 \%$ & $27,40 \%$ & $38,36 \%$ & $5,48 \%$ & $1,37 \%$ & $2,74 \%$ \\
\hline \multirow{2}{*}{ Distribuidores } & $f_{i}$ & 10 & 15 & 41 & 2 & 1 & 4 \\
\hline & $\%$ & $13,70 \%$ & $20,55 \%$ & $56,16 \%$ & $2,74 \%$ & $1,37 \%$ & $5,48 \%$ \\
\hline \multirow{2}{*}{ Proveedores } & $f_{i}$ & 5 & 13 & 45 & 5 & 2 & 3 \\
\hline & $\%$ & $6,85 \%$ & $17,81 \%$ & $61,64 \%$ & $6,85 \%$ & $2,74 \%$ & $4,11 \%$ \\
\hline \multirow{2}{*}{ Clientes } & $f_{i}$ & 7 & 13 & 41 & 6 & 1 & 5 \\
\hline & $\%$ & $9,59 \%$ & $17,81 \%$ & $56,16 \%$ & $8,22 \%$ & $1,37 \%$ & $6,85 \%$ \\
\hline \multirow{2}{*}{$\begin{array}{l}\text { Administraciones } \\
\text { públicas }\end{array}$} & $f_{i}$ & 13 & 15 & 41 & 2 & 0 & 2 \\
\hline & $\%$ & $17,81 \%$ & $20,55 \%$ & $56,16 \%$ & $2,74 \%$ & $0,00 \%$ & $2,74 \%$ \\
\hline
\end{tabular}

un 60,30\%, también las entidades financieras (57,53\%), y la Administración regional $(53,53 \%)$.

Por el contrario las fuentes menos utilizadas son los Puntos de Orientación Empresarial (POE) aunque tampoco nos extraña debido a que estos centros están más orientados a las pequeñas empresas y emprendedores, pero sí resulta destacable el escaso contacto con la institución universitaria, reflejado tanto en las fuentes formales como en las fuentes informales o personales. En el caso de Extremadura la Universidad no parece haberse convertido en motor de desarrollo.

Aunque los profesionales de la información trabajan normalmente con fuentes formales, en el mundo empresarial las fuentes informales han tenido tradicionalmente una mayor importancia en la toma de decisiones.

En la literatura sobre gestión empresarial las referencias a las fuentes verbales nos indican que las que proceden del contacto con otras personas han sido tradicionalmente las más utilizadas. El citado Francis Aguilar en su obra ya clásica "Scanning the Business Environment" descubrió que los directivos daban mucha más importancia a la información que les llegaba por el contacto personal que a las fuentes impersonales. Confrontándolas, las personales representaban un $71 \%$ frente al 29\% de las impersonales, indicando la mayor dependencia de los directivos con sus redes personales ya fueran éstas privadas o pertenecientes a la organización (Aguilar, 1967). 


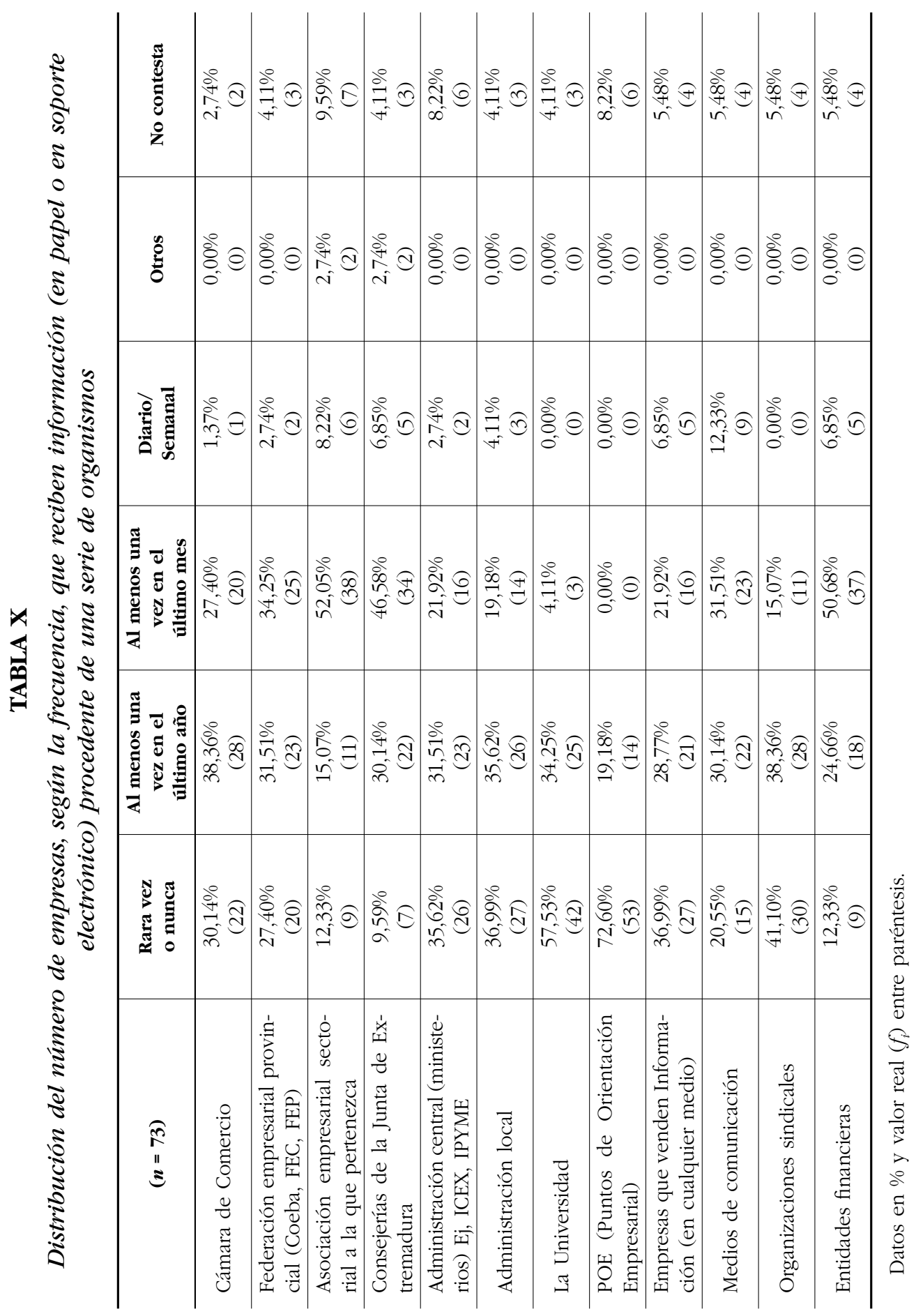




\section{TABLA XI}

Distribución del número de empresas, según la frecuencia, que recibe la información por conversaciones con personas de diversos ámbitos

\begin{tabular}{|c|c|c|c|c|c|c|}
\hline$(n=73)$ & $\begin{array}{c}\text { Rara } \\
\text { vez } \\
\text { o } \\
\text { nunca }\end{array}$ & $\begin{array}{c}\text { Al } \\
\text { menos } \\
\text { una } \\
\text { vez en } \\
\text { el } \\
\text { último } \\
\text { año }\end{array}$ & $\begin{array}{c}\text { Al } \\
\text { menos } \\
\text { una } \\
\text { vez en } \\
\text { el } \\
\text { último } \\
\text { mes }\end{array}$ & $\begin{array}{l}\text { Diario/ } \\
\text { Semanal }\end{array}$ & Otros & $\begin{array}{l}\text { No } \\
\text { contesta }\end{array}$ \\
\hline Cámara de Comercio & $\begin{array}{l}56,16 \% \\
\quad(41)\end{array}$ & $\begin{array}{l}26,03 \% \\
\quad(19)\end{array}$ & $\begin{array}{l}10,96 \% \\
\text { (8) }\end{array}$ & $\begin{array}{l}0,00 \% \\
(0)\end{array}$ & $\begin{array}{l}0,00 \% \\
(0)\end{array}$ & $\begin{array}{l}6,85 \% \\
(5)\end{array}$ \\
\hline $\begin{array}{l}\text { Federación empresarial pro- } \\
\text { vincial (Coeba, FEC, FEP) }\end{array}$ & $\begin{array}{l}47,95 \% \\
(35)\end{array}$ & $\begin{array}{l}31,51 \% \\
(23)\end{array}$ & $\begin{array}{l}16,44 \% \\
(12)\end{array}$ & $\begin{array}{l}0,00 \% \\
(0)\end{array}$ & $\begin{array}{l}0,00 \% \\
(0)\end{array}$ & $\begin{array}{l}4,11 \% \\
(3)\end{array}$ \\
\hline $\begin{array}{l}\text { Asociación empresarial sec- } \\
\text { torial a la que pertenezca }\end{array}$ & $\begin{array}{l}21,92 \% \\
\quad(16)\end{array}$ & $\begin{array}{l}23,29 \% \\
\quad(17)\end{array}$ & $\begin{array}{l}39,73 \% \\
\quad(29)\end{array}$ & $\begin{array}{l}5,48 \% \\
(4)\end{array}$ & $\begin{array}{l}0,00 \% \\
(0)\end{array}$ & $\begin{array}{l}9,59 \% \\
(7)\end{array}$ \\
\hline $\begin{array}{l}\text { Consejerías de la Junta de } \\
\text { Extremadura }\end{array}$ & $\begin{array}{l}23,29 \% \\
\quad(17)\end{array}$ & $\begin{array}{l}34,25 \% \\
\quad(25)\end{array}$ & $\begin{array}{l}28,77 \% \\
\quad(21)\end{array}$ & $\begin{array}{l}8,22 \% \\
(6)\end{array}$ & $\begin{array}{l}0,00 \% \\
(0)\end{array}$ & $\begin{array}{l}5,48 \% \\
(4)\end{array}$ \\
\hline $\begin{array}{l}\text { Administración central. } \\
\text { Ej, ICEX, IPYME }\end{array}$ & $\begin{array}{l}45,21 \% \\
(33)\end{array}$ & $\begin{array}{l}27,40 \% \\
\quad(20)\end{array}$ & $\begin{array}{c}17,81 \% \\
(13)\end{array}$ & $\begin{array}{l}4,11 \% \\
(3)\end{array}$ & $\begin{array}{l}0,00 \% \\
(0)\end{array}$ & $\begin{array}{l}5,48 \% \\
(4)\end{array}$ \\
\hline Administración local & $\begin{array}{l}38,36 \% \\
(28) \\
\end{array}$ & $\begin{array}{l}32,88 \% \\
(24)\end{array}$ & $\begin{array}{l}20,55 \% \\
(15)\end{array}$ & $\begin{array}{l}2,74 \% \\
(2)\end{array}$ & $\begin{array}{l}0,00 \% \\
(0)\end{array}$ & $\begin{array}{l}5,48 \% \\
(4)\end{array}$ \\
\hline La Universidad & $\begin{array}{c}67,12 \% \\
(49)\end{array}$ & $\begin{array}{l}20,55 \% \\
(15)\end{array}$ & $\begin{array}{l}6,85 \% \\
(5)\end{array}$ & $\begin{array}{l}0,00 \% \\
(0)\end{array}$ & $\begin{array}{l}0,00 \% \\
(0)\end{array}$ & $\begin{array}{l}5,48 \% \\
(4)\end{array}$ \\
\hline $\begin{array}{l}\text { POE (Puntos de Orientación } \\
\text { Empresarial) }\end{array}$ & $\begin{array}{l}79,45 \% \\
(58)\end{array}$ & $\begin{array}{l}9,59 \% \\
(7)\end{array}$ & $\begin{array}{c}0,00 \% \\
(0)\end{array}$ & $\begin{array}{l}0,00 \% \\
(0)\end{array}$ & $\begin{array}{l}0,00 \% \\
(0)\end{array}$ & $\begin{array}{c}10,96 \% \\
(8)\end{array}$ \\
\hline $\begin{array}{l}\text { Empresas que venden infor- } \\
\text { mación (en cualquier medio) }\end{array}$ & $\begin{array}{l}54,79 \% \\
(40)\end{array}$ & $\begin{array}{l}23,29 \% \\
(17)\end{array}$ & $\begin{array}{l}12,33 \% \\
(9)\end{array}$ & $\begin{array}{l}1,37 \% \\
(1)\end{array}$ & $\begin{array}{c}0,00 \% \\
(0)\end{array}$ & $\begin{array}{c}8,22 \% \\
(6)\end{array}$ \\
\hline Medios de comunicación & $\begin{array}{c}39,73 \% \\
(29)\end{array}$ & $\begin{array}{c}30,14 \% \\
(22)\end{array}$ & $\begin{array}{c}21,92 \% \\
\quad(16)\end{array}$ & $\begin{array}{c}1,37 \% \\
\text { (1) }\end{array}$ & $\begin{array}{c}0,00 \% \\
(0)\end{array}$ & $\begin{array}{c}6,85 \% \\
(5)\end{array}$ \\
\hline Organizaciones sindicales & $\begin{array}{c}49,32 \% \\
(36)\end{array}$ & $\begin{array}{c}28,77 \% \\
(21)\end{array}$ & $\begin{array}{c}10,96 \% \\
(8)\end{array}$ & $\begin{array}{c}0,00 \% \\
(0)\end{array}$ & $\begin{array}{c}0,00 \% \\
(0)\end{array}$ & $\begin{array}{c}10,96 \% \\
(8)\end{array}$ \\
\hline Entidades financieras & $\begin{array}{c}10,96 \% \\
(8)\end{array}$ & $\begin{array}{c}27,40 \% \\
(20)\end{array}$ & $\begin{array}{c}46,58 \% \\
(34)\end{array}$ & $\begin{array}{c}6,85 \% \\
(5)\end{array}$ & $\begin{array}{c}1,37 \% \\
(1)\end{array}$ & $\begin{array}{c}6,85 \% \\
(5)\end{array}$ \\
\hline Clientes & $\begin{array}{c}5,48 \% \\
(4) \\
\end{array}$ & $\begin{array}{c}17,81 \% \\
(13) \\
\end{array}$ & $\begin{array}{c}57,53 \% \\
(42) \\
\end{array}$ & $\begin{array}{c}10,96 \% \\
(8)\end{array}$ & $\begin{array}{c}1,37 \% \\
(1)\end{array}$ & $\begin{array}{c}6,85 \% \\
(5)\end{array}$ \\
\hline Proveedores & $\begin{array}{c}6,85 \% \\
(5)\end{array}$ & $\begin{array}{c}15,07 \% \\
(11)\end{array}$ & $\begin{array}{c}60,27 \% \\
(44)\end{array}$ & $\begin{array}{c}9,59 \% \\
(7)\end{array}$ & $\begin{array}{c}1,37 \% \\
(1)\end{array}$ & $\begin{array}{c}6,85 \% \\
(5)\end{array}$ \\
\hline Distribuidores & $\begin{array}{c}12,33 \% \\
\text { (9) }\end{array}$ & $\begin{array}{c}17,81 \% \\
(13)\end{array}$ & $\begin{array}{c}50,68 \% \\
(37) \\
\end{array}$ & $\begin{array}{c}9,59 \% \\
(7)\end{array}$ & $\begin{array}{c}1,37 \% \\
\text { (1) }\end{array}$ & $\begin{array}{c}8,22 \% \\
(6)\end{array}$ \\
\hline Colegas del mismo sector & $\begin{array}{c}8,22 \% \\
(6)\end{array}$ & $\begin{array}{c}23,29 \% \\
(17)\end{array}$ & $\begin{array}{c}54,79 \% \\
(40)\end{array}$ & $\begin{array}{c}5,48 \% \\
(4)\end{array}$ & $\begin{array}{c}1,37 \% \\
(1)\end{array}$ & $\begin{array}{c}6,85 \% \\
(5)\end{array}$ \\
\hline Ferias comerciales & $\begin{array}{c}20,55 \% \\
(15)\end{array}$ & $\begin{array}{c}47,95 \% \\
(35)\end{array}$ & $\begin{array}{c}20,55 \% \\
(15)\end{array}$ & $\begin{array}{c}4,11 \% \\
(3)\end{array}$ & $\begin{array}{c}0,00 \% \\
(0)\end{array}$ & $\begin{array}{c}6,85 \% \\
(5)\end{array}$ \\
\hline
\end{tabular}

Datos en \% y valor real $\left(f_{i}\right)$ entre paréntesis. 
El clásico estudio de Henry Mintzberg "La naturaleza del trabajo directivo" que estudia el comportamiento de cinco directivos, extrae como conclusión más clara como las fuentes personales se convierten para el directivo en la fuente privilegiada para la toma de decisiones, de tal manera que: "la fuerte atracción hacia los medios verbales de la que dieron prueba los directivos se revela como el hallazgo más significativo sobre los medios de comunicación utilizados" (Mintzberg, 1983). Si la interacción verbal suponía el 78\% del tiempo de los directivos encuestados, también lo era el $67 \%$ de sus actividades. Lo que el estudio de Mintzberg también dejaba claro era la existencia de una red de contactos. Para este autor "los contactos de los directores generales de su estudio incluían clientes, socios, proveedores, compañeros que gestionaban sus propias organizaciones, funcionarios de organismos sectoriales y gubernamentales", y otras personas del exterior de la empresa.

Observando la Tabla XI, podemos concluir, si sumamos las columnas «diario/ semanal" y "al menos una vez en el último mes", que las conversaciones con personas que pertenecen al entorno externo más inmediato, como las de entidades financieras $(53,43 \%)$, clientes $(68,49 \%)$, proveedores $(69,86 \%)$, distribuidores $(60,27 \%)$, colegas del mismo sector $(60,27 \%)$, tienen un mayor peso en general que las fuentes verbales que proceden de resto de los ámbitos. Lo que coincide con el peso que las fuentes de este entorno reflejan en los resultados de la Tabla IX.

\section{Conclusiones}

La gestión de la información es un proceso intrínseco a cualquier empresa, en tanto que como organización genera datos por su propio funcionamiento interno, y ello deriva en un proceso de tratamiento de esos datos y su correspondiente documentación. A su vez, como entidad que se encuentra en un entorno, necesita de información para tomar decisiones, de tal manera que se haga de una manera consciente a través del establecimiento de políticas explicitadas o no, todo gerente, administrador o equipo de dirección toman decisiones en base a informaciones que les pueden llegar por múltiples canales, ya sean esos canales formales o informales.

Este trabajo ha buscado indagar en ese entorno informativo, en las prácticas internas en gestión de la información y del conocimiento, si las hubiera, en el conocimiento de los entornos externos, que en la teoría de la gestión empresarial se denominan como entorno externo remoto y entorno externo inmediato, o en el origen de las fuentes externas, ya procedan de canales formales como informales.

De las 144 empresas seleccionadas, para llevar a cabo la encuesta, 73 completaron el cuestionario, y aunque el índice de respuesta es elevado para un estudio de estas características (un 50,69\%) las conclusiones hay que tomarlas con la prudencia que debe de caracterizar a dicho porcentaje. 
Destaca que un porcentaje significativo de empresas, casi el 70\%, no dispone de biblioteca y/o unidad de información y documentación. De cualquier forma a la pregunta de si la empresa disponía de alguna persona o unidad dedicada a las tareas de recopilación, organización y análisis de la información relevante (es decir tareas de gestión de la información) tanto con dedicación exclusiva como sin dedicación exclusiva, sólo un 2,74\% (dos empresas) respondieron que sí disponían de personas con dedicación exclusiva. Sin dedicación exclusiva el porcentaje de empresas era mayor, un 45,21\%. Otra cuestión relacionada con las anteriores preguntaba sobre si las empresas disponían de algún mecanismo estructurado en forma de departamento o persona encargado de capturar información externa para la toma de decisiones. Destaca que en torno a la mitad respondiera que sí. Pudiera deducirse que existe un creciente interés en capturar información del entorno exterior, por parte de las empresas, aunque esta captura no se haga con personal dedicado exclusivamente a tal fin.

Las fuentes de información impresas o en soporte electrónico (Tabla VII) más utilizadas para la toma de decisiones son la prensa, legislación, normativas y convenios colectivos, y los catálogos de productos y/o catálogos de ferias comerciales; sin embargo, destaca que la tipología señalada como directorios de empresas, productos e informes comerciales de otras empresas con la inclusión de datos de impagados sea de las menos demandadas.

Las Tablas VIII y IX nos presentan los resultados de uso de la información procedente del medio ambiente externo remoto y del medio ambiente externo inmediato. Si sumamos las cifras de las opciones «al menos una vez en el último mes" y "diario/semanal" que harían referencia a un uso más continuado de los entornos referidos encontramos los siguientes resultados respecto al entorno remoto: sociedad y cultura 8,22\%; economía, 50,69\%; tecnología, 57,53\%; y política y legislación, 58,9\%. Respecto al entorno inmediato las cifras son las siguientes: competidores, 43,84\%; distribuidores, 58,9\%; proveedores, 68,49\%; clientes, 64,38\%; y Administraciones públicas, 58,9\%. Así proveedores y clientes se convierten en las fuentes más utilizadas.

En cuanto a la información que llega a la empresa, procedente de entidades, organismos, etc., y uniendo las opciones "al menos una vez en el último mes" y "diario/semanal" en lo que a documentos (en papel o soporte electrónico) se refiere, tenemos un ranking que lideran las asociaciones empresariales, en un porcentaje alto $(60,27 \%)$, seguido de las entidades financieras $(57,53 \%)$ y de la administración regional $(53,43 \%)$. Lo cual presenta resultados muy similares al estudio de Giménez y Toledo para empresas de la Comunidad de Madrid (Giménez Toledo, 2004). Y respecto a los canales informales (y uniendo las mismas opciones que para el caso anterior) encontramos una relación que encabezan los proveedores $(69,86 \%)$, seguida de los clientes $(68,49 \%)$, distribuidores y colegas del mismo sector (con un 60,27\%), las entidades financieras $(53,43 \%$ ) y las asociaciones empresariales sectoriales $(45,21 \%)$. Por el contrario, destaca la poca sintonía con la universidad o las cámaras de comercio. 


\section{Bibliografía}

Aguilar, F. J. (1967): Scanning the Business Environment. New York: Macmillan.

Auster, E., y Choo, C. (1994). How senior managers acquire and use information in environment scanning, Information Processing \& Management, vol. 30 (5), 607-618.

Cornella, A., y Flores, A. (2007): La alquimia de la innovación. Barcelona: Deusto.

Fernández Bajon, M. T. (1998). La profesión de documentalista: apuntes para una reflexión, Boletín de la ANABAD, vol. 49 (2), 295-307.

GIA International (2005): Competitive Intelligence in Large Companies. Global Study 2005 [web]. [Consulta: 06-06-2008]. Disponible en Web: http://www.globalintelligence.com/ news/whitepapers/.

GIA International (2007): Market Intelligence in Large Companies. Global Study 2007 [web]. [Consulta: 06-06-2008]. Disponible en Web: http://www.globalintelligence.com/news/ whitepapers/.

Giménez Toledo, E., y Román Román, A. (2004): Productores de información interesantes para las empresas. Resultados de una encuesta sobre consumo de información a empresas de la Comunidad de Madrid, Revista Española de Documentación Científica, vol. 27 (1), 73-92.

Glynm, K.; Koening, E. D., y Michael, E. (1995): Small business and information technology, Annual Review of Information Science and Technology (ARIST), vol. 30, 251280.

Gold, D. (1999): The information professional: an asset for competitive intelligence, FID Review, vol. 1 (4/5), 77-82.

INE. (2006): Estadistica de I+D 2006. Madrid: INE.

Ivancevich, John M.; Lorenzi, P.; Skinner, Steven J., y Crosby, Philip B. (1997): Gestión, calidad y competitividad. Madrid: McGraw-Hill.

Keegan, W. J. (1968): The adquisition of global information, Information Management Review, vol. 8 (1), 54-56.

Latorre Zacarés, J.; Salinas Pardo, C.; García Pardo, S., y Estrada Luna, A. (2007): La vigilancia tecnológica como un aspecto de la innovación en la gestión de las organizaciones: caso práctico. En X Jornadas Españolas de Documentación. Santiago de Compostela, mayo, págs. 181-186.

Lenz, R. T., y Engledow, J. L. (1986): Environment analysis units and strategic decisionmaking: a field study of selected ëleading-edgeí corporations, Strategic Management Journal, vol. 7, 69-89.

Mintzberg, H. (1983): La naturaleza del trabajo directivo. Barcelona: Ariel.

Muñoz Cañavate, A. (2007): La enseñanza de la información para la empresa en la universidad española. Reflexiones para una orientación académica y profesional de la disciplina, Documentación de las Ciencias de la Información, vol. 30, 253-274.

Muñoz Cañavate, A., y Chain Navarro, C. (2006): Una aproximación a los estudios sobre información para la empresa en las Universidades españolas. En: VII Encuentro de EDIBCIC. Marilia (Sao Paulo) Brasil, noviembre, págs. 223-242.

Muñoz Cañavate, A., y Hípola, P. (2008): Business information through Spain's Chambers of Commerce: Meeting business needs. Business Information Review, vol. 25 (4), 224-229. 
Muñoz Cañavate, A., y Hípola Ruíz, P. (2009): Information studies for the business sector in Spanish universities, Information for Education, vol. 26 (3-4), 191-201.

Postigo, J. (2001): La inteligencia competitiva en España: un encuentro sobre su utilización por parte de las empresas exportadoras, El Profesional de la Información, vol. 10 (10), 4-11.

Sanz, E., y Rubio, L. (1993): Necesidades de información en las empresas: estudio de un caso, Revista Española de Documentación Científica, vol. 16 (3), 229-236.

Tena Millán, J., y Comai, A. (2004): La Inteligencia Competitiva en las Multinacionales Catalanas. Barcelona: Emecon Consultores.

Tena Millán, J., y Comai, A. (comp.) (2006): Inteligencia Competitiva y Vigilancia Tecnológica. Experiencias de Implantación en España y Latinoamérica. Barcelona; EMECOM.

Vázquez Valero, M., y Velayos Huerta, A. (1999): El consumo de información científico-técnica por las empresas, Anales de Documentación, vol. 2, 63-83.

Xianzhong, M. Xu.; Roland Kaye, G., y Duan, Y. (2003): UK executive vision on business environment for information scanning. A cross industry study, Information \& Management, vol. 40, 381-389.

Zamorano Martínez, L. (2005): La información empresarial en el marco de las políticas de promoción industrial de la Junta de Extremadura. En: Muñoz Cañavate, A. (coord.). La información empresarial en Extremadura. Badajoz: Diputación Provincial, 2005, 115-158. 


\section{ANEXO}

Cuestionario completo enviado a las empresas

\section{BLOQUE. GESTIÓN DE RECURSOS DE INFORMACIÓN Y DEL CONOCIMIENTO EN LA EMPRESA}

1. ¿Cuenta su empresa con biblioteca o unidad de información/documentación?

Sí $\square \quad$ No

2. ¿Cuenta su empresa con alguna persona o unidad que se dedique a las tareas de recopilación, organización y análisis de información relevante para la empresa, sin dedicación en exclusiva a estas tareas?

Sí $\square \quad$ No

Si la tiene especifique su cargo

3. ¿Cuenta su empresa con una persona o unidad dedicada a las tareas de recopilación, organización y análisis de información relevante para la empresa, con dedicación exclusiva?

\section{Sí $\square \quad$ No $\square$}

Si la tiene especifique su cargo

4. ¿Recibe en su empresa revistas profesionales de su sector y prensa general y/o especializada en economía, finanzas y mercado?

Sí $\square \quad$ No

5. ¿Está suscrito o registrado en bases de datos (CdRom, por Internet, etc.) de interés para su empresa? (las suscripciones pueden ser gratuitas).

Sí $\square \quad$ No $\square$

6. Si ha respondido sí a la pregunta anterior, por favor especifique el tipo de información: (Marque tantos como sea necesario).

- Directorios de empresas y/o productos

- Informes comerciales (incluye impagados) de otras empresas

- Indicadores del entorno (estadísticas, informes económicos realizados por Administraciones, bancos, etc., o magnitudes macroeconómicas)

- Bases de datos de prensa (general o especializada)

- Mercados financieros (de crédito, renta fija, variable, futuros, etc.)

- Ayudas 
- Concursos público

- Informes sectoriales/estudios de mercado

- Legislación y/o jurisprudencia

- Normativas sectoriales (convenios colectivos)

- Normas de producción (Ej., ISO, Aenor)

- Patentes, dibujos/diseños industriales, o marcas

7. ¿Dispone de un mecanismo estructurado (algún departamento o persona) encargado de capturar información externa para la toma de decisiones? Por ejemplo, cuando hay que comprar un informe comercial sobre clientes o competidores, o conseguir información sobre ayudas públicas o concursos públicos (estos casos son sólo algunos ejemplos)

\section{Sí $\square \quad$ No $\square$}

8. ¿Ha puesto en marcha, alguna vez, un proceso de auditoría de información dentro de su empresa: conocer quien utiliza la inf., donde se encuentra, cómo se utiliza, cómo está estructurada y en qué soportes. Nos referimos a los distintos depósitos de información: las bases de datos internas, las externas de pago, los archivos de empresa.

\section{Sí $\square \quad$ No $\square$}

9. ¿Dispone de alguna forma estructurada para beneficiarse y registrar en una memoria de la empresa los conocimientos de los empleados?

\section{Sí $\square \quad$ No $\square$}

10. Si sus empleados tienen información sobre sus competidores ¿existe algún medio, en su organización para compartir esa información?

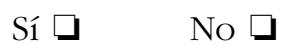




\section{BLOQUE. EL CONJUNTO DE INFORMACIÓN QUE SE PUEDE NECESITAR PARA LA TOMA DE DECISIONES}

¿Obtiene usted o el personal de su empresa información sobre los siguientes aspectos?

Marque con una $\mathrm{x}$. Incluye cualquier sistema o soporte (personas, material impreso, Internet, etc.)

\begin{tabular}{|c|c|c|c|c|}
\hline & $\begin{array}{l}\text { Rara vez o } \\
\text { nunca }\end{array}$ & $\begin{array}{c}\text { Al menos } \\
\text { una vez en el } \\
\text { último año }\end{array}$ & $\begin{array}{c}\text { Al menos } \\
\text { una vez en el } \\
\text { último mes }\end{array}$ & $\begin{array}{l}\text { Otros: } \\
\text { especifique }\end{array}$ \\
\hline \multicolumn{5}{|c|}{ Medio Ambiente Externo (Entorno remoto) } \\
\hline \multicolumn{5}{|l|}{$\begin{array}{l}\text { Sociedad y Cultura (roles } \\
\text { cambiantes de la mujer, es- } \\
\text { tilo de vida, planificación } \\
\text { familiar, etc.) }\end{array}$} \\
\hline \multicolumn{5}{|l|}{$\begin{array}{l}\text { Economía (datos macroeco- } \\
\text { nómicos; tipos de interés, } \\
\text { fuentes y costes de la ener- } \\
\text { gía, etc) }\end{array}$} \\
\hline \multicolumn{5}{|l|}{$\begin{array}{l}\text { Tecnología (nuevos produc- } \\
\text { tos, I+D en la industria, tec- } \\
\text { nología informática, etc) }\end{array}$} \\
\hline \multicolumn{5}{|l|}{$\begin{array}{l}\text { Política, Legislación (regula- } \\
\text { ciones comerciales, deduc- } \\
\text { ción impuestos, etc) }\end{array}$} \\
\hline \multicolumn{5}{|c|}{ Medio Ambiente Externo (Entorno inmediato) } \\
\hline \multicolumn{5}{|l|}{$\begin{array}{l}\text { Competidores (Ej., empre- } \\
\text { sas, sus productos) }\end{array}$} \\
\hline \multicolumn{5}{|l|}{ Distribuidores } \\
\hline \multicolumn{5}{|l|}{$\begin{array}{l}\text { Proveedores (empresas, } \\
\text { precios) }\end{array}$} \\
\hline \multicolumn{5}{|l|}{ Clientes (sus intereses) } \\
\hline \multicolumn{5}{|l|}{ Acreedores } \\
\hline Administraciones Públicas & & & & \\
\hline
\end{tabular}




\section{BLOQUE. LOS CONTENIDOS INFORMATIVOS PARA LA TOMA DE DECISIONES}

1. ¿Con qué frecuencia consulta ud. o los empleados de su empresa los siguientes tipos de información en su trabajo para la toma de decisiones? (Se incluyen información en cualquier soporte: impreso, Internet, etc.)

\begin{tabular}{|c|c|c|c|c|}
\hline Fuentes formales & $\begin{array}{c}\text { Rara vez o } \\
\text { nunca }\end{array}$ & $\begin{array}{c}\text { Al menos } \\
\text { una vez en el } \\
\text { último año }\end{array}$ & $\begin{array}{c}\text { Al menos } \\
\text { una vez en el } \\
\text { último mes }\end{array}$ & $\begin{array}{l}\text { Otros: } \\
\text { especifique }\end{array}$ \\
\hline $\begin{array}{l}\text { - Directorios de empresas } \\
\text { y/o productos (incluye im- } \\
\text { pagados) }\end{array}$ & & & & \\
\hline $\begin{array}{l}\text { - Informes comerciales de } \\
\text { otras empresas }\end{array}$ & & & & \\
\hline $\begin{array}{l}\text { - Indicadores del entorno } \\
\text { (estadísticas, informes eco- } \\
\text { nómicos realizados por Ad- } \\
\text { ministraciones, bancos, } \\
\text { etc., o magnitudes ma- } \\
\text { croeconómicas) }\end{array}$ & & & & \\
\hline $\begin{array}{l}\text { - Prensa (general o especia- } \\
\text { lizada) }\end{array}$ & & & & \\
\hline - Mercados financieros & & & & \\
\hline - Ayudas & & & & \\
\hline - Concursos públicos & & & & \\
\hline $\begin{array}{l}\text { - Informes sectoriales/estu- } \\
\text { dios de mercado }\end{array}$ & & & & \\
\hline $\begin{array}{l}\text { - Legislación y/o jurispru- } \\
\text { dencia }\end{array}$ & & & & \\
\hline $\begin{array}{l}\text { - Patentes, dibujos/diseños } \\
\text { industriales, o marcas }\end{array}$ & & & & \\
\hline $\begin{array}{l}\text { - Revistas, referencias biblio- } \\
\text { gráficas }\end{array}$ & & & & \\
\hline - Conferencias, congresos & & & & \\
\hline $\begin{array}{l}\text { - Catálogos de productos } \\
\text { y/o catálogos de ferias } \\
\text { comerciales }\end{array}$ & & & & \\
\hline
\end{tabular}




\section{BLOQUE. SOPORTES Y MEDIOS POR LOS QUE LE LLEGA INFORMACIÓN}

1. Puntúe de 0 a 5 el uso de los siguientes medios para adquirir información ( 0 cuando nunca se utilice y 5 cuando sea un uso continuado)

Papel

Teléfono

Fax

Internet (correo electrónico)

Internet (servicios web)

Conversaciones presenciales

Otros:
De 0 a 5

De 0 a 5

De 0 a 5

De 0 a 5

De 0 a 5

De 0 a 5

De 0 a 5

2. ¿Qué tipo de fuentes considera que son más importantes para la toma de decisiones?

Fuentes en algún tipo de soporte: papel, Internet, etc.

Las personas a través de conversaciones

Las dos anteriores por igual

3. Puntúe de 0 a 5 la importancia de la procedencia de la información para la toma de decisiones:

Conversaciones dentro de la empresa

Conversaciones fuera de la empresa

De 0 a 5

Documentos (en papel o electrónico)

que se generan dentro de la empresa

Documentos (en papel o electrónicos)

que se generan fuera de la empresa

De 0 a 5

De 0 a 5

De 0 a 5 


\section{BLOQUE. ORIGEN DE LA INFORMACIÓN QUE NECESITA: FUENTES EXTERNAS (LUGAR DE DONDE PROCEDE LA INFORMACIÓN)}

1. ¿Consulta o le llegan información a través de los siguientes organismos?

\begin{tabular}{l|l|l|l|l}
\hline & $\begin{array}{c}\text { Rara vez o } \\
\text { nunca }\end{array}$ & $\begin{array}{c}\text { Al menos } \\
\text { una vez en } \\
\text { el último } \\
\text { año }\end{array}$ & $\begin{array}{c}\text { Al menos } \\
\text { una vez en } \\
\text { éltimo } \\
\text { mes }\end{array}$ & $\begin{array}{c}\text { Otros: } \\
\text { especifique }\end{array}$ \\
\hline Cámara de Comercio & & & \\
\hline $\begin{array}{l}\text { Federación empresarial provincial } \\
\text { (Coeba, FEC, FEP) }\end{array}$ & & & \\
\hline $\begin{array}{l}\text { Asociación empresarial sectorial a } \\
\text { la que pertenezca }\end{array}$ & & & \\
\hline $\begin{array}{l}\text { Consejerías de la Junta de Extrema- } \\
\text { dura }\end{array}$ & & & \\
\hline $\begin{array}{l}\text { Administración central (ministe- } \\
\text { rios) Ej. Instituto Español de Co- } \\
\text { mercio Exterior D. G. de Política } \\
\text { de la Pyme }\end{array}$ & & & & \\
\hline Administración local & & & & \\
\hline $\begin{array}{l}\text { La Universidad } \\
\text { POE (Puntos de Orientación Em- } \\
\text { presarial) }\end{array}$ & & & & \\
\hline $\begin{array}{l}\text { Empresas que venden información } \\
\text { medio: Internet, CdRom) }\end{array}$ & & & & \\
\hline $\begin{array}{l}\text { Medios de comunicación } \\
\text { Organizaciones sindicales }\end{array}$ & & & & \\
\hline Entidades financieras & & & \\
\hline
\end{tabular}


2. ¿Consulta o le llegan información a través de conversaciones con personas que pertenezcan a los siguientes organismos?

\begin{tabular}{|c|c|c|c|c|}
\hline & $\begin{array}{l}\text { Rara vez o } \\
\text { nunca }\end{array}$ & $\begin{array}{l}\text { Al menos } \\
\text { una vez en } \\
\text { el último } \\
\text { año }\end{array}$ & $\begin{array}{l}\text { Al menos } \\
\text { una vez en } \\
\text { el último } \\
\text { mes }\end{array}$ & $\begin{array}{l}\text { Otros: } \\
\text { especifique }\end{array}$ \\
\hline \multicolumn{5}{|l|}{ Cámara de Comercio } \\
\hline \multicolumn{5}{|l|}{$\begin{array}{l}\text { Federación empresarial provin- } \\
\text { cial (Coeba, FEC, FEP) }\end{array}$} \\
\hline \multicolumn{5}{|l|}{$\begin{array}{l}\text { Asociación empresarial secto- } \\
\text { rial a la que pertenezca }\end{array}$} \\
\hline \multicolumn{5}{|l|}{$\begin{array}{l}\text { Consejerías de la Junta de Ex- } \\
\text { tremadura }\end{array}$} \\
\hline \multicolumn{5}{|l|}{$\begin{array}{l}\text { Administración central (ministe- } \\
\text { rios) }\end{array}$} \\
\hline \multicolumn{5}{|l|}{ Administración local } \\
\hline \multicolumn{5}{|l|}{ La Universidad } \\
\hline \multicolumn{5}{|l|}{$\begin{array}{l}\text { POE (Puntos de Orientación } \\
\text { Empresarial) }\end{array}$} \\
\hline \multicolumn{5}{|l|}{$\begin{array}{l}\text { Empresas que venden informa- } \\
\text { ción (Ej. Bases de datos por } \\
\text { cualquier medio: Internet, } \\
\text { CdRom) }\end{array}$} \\
\hline \multicolumn{5}{|l|}{ Medios de comunicación } \\
\hline \multicolumn{5}{|l|}{ Organizaciones sindicales } \\
\hline \multicolumn{5}{|l|}{$\begin{array}{l}\text { Conversaciones con empleados } \\
\text { de entidades financieras }\end{array}$} \\
\hline \multicolumn{5}{|l|}{ Conversaciones con clientes } \\
\hline \multicolumn{5}{|l|}{$\begin{array}{l}\text { Conversaciones con provee- } \\
\text { dores }\end{array}$} \\
\hline \multicolumn{5}{|l|}{$\begin{array}{l}\text { Conversaciones con distribui- } \\
\text { dores }\end{array}$} \\
\hline \multicolumn{5}{|l|}{$\begin{array}{l}\text { Conversaciones con colegas del } \\
\text { mismo sector }\end{array}$} \\
\hline $\begin{array}{l}\text { Conversaciones en ferias co- } \\
\text { merciales }\end{array}$ & & & & \\
\hline
\end{tabular}

